\title{
Evaluation of a Rapid Culture-Based Screening Test for Detection of Methicillin resistant Staphylococcus aureus
}

\author{
BOUSHRA FWITY ${ }^{1}$, RALF LOBMANN ${ }^{2}$ and ANDREAS AMBROSCH${ }^{1 \star}$ \\ ${ }^{1}$ Institute of Laboratory Medicine and Microbiology, St.Joseph Hospital, Bremerhaven, Germany \\ ${ }^{2}$ Dept. of Endocrinology, Diabetology and Geriatrics, Stuttgart General Hospital, Germany
}

Received 22 November 2010, revised 4 May 2011, accepted 24 May 2011

\begin{abstract}
The performance of a culture based assay, BacLite ${ }^{\mathrm{mm}}$ Rapid MRSA for the rapid detection (5 hours) of methicillin resistant Staphylococcus aureus (MRSA) from specimens $(\mathrm{n}=377)$ obtained from nares, throat, wounds and perineum was investigated. Compared to culture based reference methods (chromogenic MRSA ID (bioMerieux)), selective enrichment broth, PBP2' latex agglutination (Oxoid) and VITEK 2 identification (bioMerieux), an overall sensitivity of $71 \%$ with a $82 \%$ specificity and a negative predictive value (NPV) of $95 \%$ was provided. The Baclite ${ }^{\text {Tn }}$ test is rapid and easy to use and has the advantage of a culture-based detection method for MRSA.
\end{abstract}

Ke y words: Baclite $^{\text {tix }}$ Rapid MRSA test, chromogenic agar, test evaluation

Active screening and compliance to appropriate infection control activities have been shown to play an important role in the control of MRSA (Kluytmans, 2007). Rapid diagnostic tests have the potential to make efforts even more effective. Thus, infection prevention has taken a step forward with the introduction of various tests for rapid identification of MRSA carriers and infections (Harbarth et al., 2006). However, a variety of increasingly sophisticated DNA-based tests have been developed to detect MRSA more rapidly (Francois et al., 2003; Huletsky et al., 2004). Most of these assays are based on the detection of Staphylococcus aureus specific sequences and the mecA gene. Despite the technical improvements in molecular based assays, their high cost and relatively high operator skill requirement remains obstacle to their widespread routine use. In addition, in a metaanalysis of ten studies on the effect of MRSA detection by rapid molecular screening tests compared to culture alone, no evidence was found on the effect of rapid testing on hospital-acquired MRSA infections and acquisition rate (Tacconelli et al., 2009). However, one problem might be the high sensitivity by amplification of bacterial sequences leading to an overestimation of MRSA colonization. This is assay immanent, since bacterial sequences were detected and not viable strains.

The present study describes the evaluation of a commercially available rapid culture based test - the Baclite ${ }^{\mathrm{Tw}}$ Rapid MRSA test - which has been developed to detect ciprofloxacin resistant MRSA strains within 5 hours. This new test was compared to a second generation chromogenic agar media combined with a selective enrichment broth for detection of viable MRSA. Sensitivities and specificities of the reference method were nearly that found for molecular methods (Perry et al., 2004; Reverdy et al., 2005).

The Baclite ${ }^{\text {tw }}$ Rapid MRSA test measures Adenylate Kinase (AK) activity, which is an essential house keeping enzyme found inside all cells, which regulates energy provision by catalyzing the equilibrium reaction of ATP + AMP $\rightarrow 2$ ADP. By supplying purified ADP in vitro, the reaction can be driven to generate up to thousands ATP molecules per minute. The amplified levels of ATP produced during minutes can then be measured using the bioluminescence reaction of firefly luciferase. In the present assay, AK detection is combined with selective broth enrichment, magnetic microparticle extraction and selective lyse of S. aureus to add target organism specificity. In the extraction step, paramagnetic micro-particles coupled with a mouse antiStaphylococcus aureus monoclonal antibody are used to capture MRSA. The unbound fraction is removed by washing procedures. Capture and washing occur as automated steps inside the automated wash module. In the lyses step, a reagent containing lysostaphin and $\mathrm{ADP}$ is added and the S. aureus in the sample lysed to release AK. The AK then catalyses the conversion of ADP to ATP (Squirrel et al., 2002).

\footnotetext{
* Corresponding author: A. Ambrosch, Institute of Laboratory Medicine and Microbiology, Wienerstr. 1, 27568 Bremerhaven;
} phone: 0049-471-4805539; fax: 0049-471-4805668; e-mail dr.ambrosch@josephhospital.de 


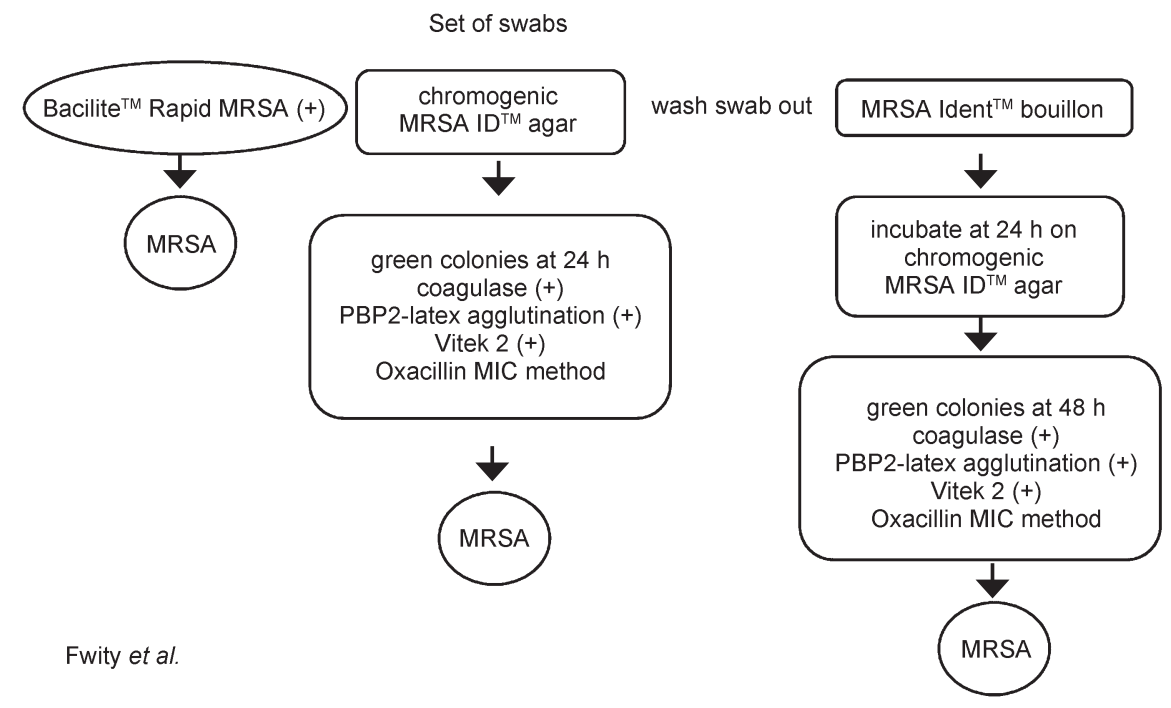

Fig. 1. The diagnostic algorithm for the identification of MRSA: inoculation of swabs to Baclite ${ }^{\mathrm{m}}$ Rapid MRSA assay compared to chromogenic MRSA ID agar and the subsequent procedure.

Up to now, only a few studies were done with the Baclite $^{\text {tn }}$ Rapid MRSA assay. One study has dealt with its reliability to discriminate MRSA from a well characterized S. aureus strain collection (Von Eiff et al., 2007), and two others analyzing the clinical performance for nasal and groin swabs (O'Hara et al., 2007; Johnson et al., 2006). However, no data exists on the overall sensitivity and specificity of this new assay for the detection of MRSA from swabs obtained from the perineum and the side of chronic wound infection. This is of interest, since national hygiene guidelines for the surveillance of MRSA also provide swabbing of the perineum and chronic ulcers as potential sides of MRSA colonization. These sides have the diagnostic disadvantage of a high density of multiple microbial bystanders potentially interfering with the sensitivity or specificity of culture based MRSA tests.

In the present study, swabs were collected from the anterior nares $(\mathrm{n}=143)$, the throat $(43)$, the perineum (113) and chronic ulcers (78). All swabs (cotton swabs with non-charcoal Amies transport medium, bioMerieux, La Balme Les Grottes, France) were taken as part of routine screening for MRSA colonization or infection according to the German national guideline for the infection control policies (www.rki.de). Specimens were transported rapidly and tested on the same day of sampling. Two single swabs from each site were elected, one for the Baclite ${ }^{\text {mit }}$ Rapid MRSA assay and the second one for the culture reference methods.

A flow diagram of the processing protocol for swabs is depicted in Fig. 1: one swab sample was first spread on chromogenic MRSA ID ${ }^{\text {tw }}$ medium (bioMerieux) and then washed out in a selective MRSA-Ident ${ }^{\mathrm{tm}}$ bouillon containing cefoxitine/sulbactam (Heipha). After incubation at $37^{\circ} \mathrm{C}$ for $18-24$ hours, green colonies on MRSA agar were regarded as presumptive $S$. aureus isolates.
The selective broth was plated on chromogenic MRSA ID and then incubated for an addition $24 \mathrm{~h}$ to increase sensitivity for detection of MRSA. S. aureus isolates were confirmed and identified using the PBP2' latex agglutination test (Oxoid), a coagulase test (Oxoid) and the Vitek 2 identification and resistance testing system (GP card and AST-P554 card, bioMerieux).

The second set of swabs was processed by the Baclite $^{\mathrm{Tm}}$ Rapid method according to the manufactures instructions. MRSA plates were inoculated first followed by the Baclite ${ }^{\mathrm{Tw}}$ Rapid MRSA assay within 2 hours to avoid processing delay. Positive and negative control strains (MRSA and MSSA) were included as procedural controls in each run. Swab samples were vortexed in the proprietary Baclite ${ }^{\mathrm{m} x}$ selective broth (containing ciprofloxacin $(6 \mathrm{mg} / \mathrm{L})$ for two times and followed by an incubation period for $2.5 \mathrm{~h}$ at $37^{\circ} \mathrm{C}$. Before the assay procedure was continued, the selective broth was subcultured on MRSA ID. This was done to evaluate the selectivity of the broth and to confirm the results of the rapid MRSA assay. However, following the manufactures instructions, MRSA were captured and washed in the Baclite ${ }^{\mathrm{Tx}}$ sample processor. The bound fraction was resuspended in the selective broth and aliquots of each sample were placed into two adjacent wells of a 96 well assay plate. One well of each sample was used to determine a baseline signal in the Baclite ${ }^{\mathrm{Tm}}$ reader. After a further incubation period of 2 hours at $37^{\circ} \mathrm{C}$, the second well for each sample was processed in the same way. The result was determined by subtraction the second from the first result and scored automatically as positive or negative to a software embedded algorithm.

Of the 377 surveillance specimens, S. aureus MRSA was isolated and confirmed from 49 samples by the reference methods. By the Baclite ${ }^{\mathrm{Tw}}$ MRSA test, 89 of the samples were positive and 288 were detected as nega- 
Table I

Comparison of Baclite ${ }^{\mathrm{TM}}$ Rapid MRSA test results with those obtained by reference methods.

\begin{tabular}{|l|c|c|c|c|c|c|}
\hline \multicolumn{1}{|c|}{$\begin{array}{c}\text { Baclite } \\
\text { methods }\end{array}$} & $\begin{array}{c}\text { No of positive } \\
\text { samples }\end{array}$ & $\begin{array}{c}\text { No of negative } \\
\text { samples }\end{array}$ & $\begin{array}{c}\text { Sensitivity } \\
(\%)\end{array}$ & $\begin{array}{c}\text { Specificity } \\
(\%)\end{array}$ & $\begin{array}{c}\text { PPV } \\
(\%)\end{array}$ & $\begin{array}{c}\text { NPV } \\
(\%)\end{array}$ \\
\hline All swabs (n=377) & $89 / 49$ & $288 / 328$ & 71 & 82 & 36 & 95 \\
\hline From nares / throat $(\mathrm{n}=186)$ & $38 / 27$ & $148 / 159$ & 70 & 84 & 43 & 93 \\
\hline From chronic wounds $(\mathrm{n}=78)$ & $25 / 13$ & $53 / 65$ & 69 & 74 & 33 & 94 \\
\hline Perineum (n=113) & $26 / 9$ & $87 / 104$ & 77 & 81 & 27 & 98 \\
\hline
\end{tabular}

PPV - positive predictive value; NPV - negative predictive value

tive. Since 35 out of the 49 confirmed MRSA samples were detected by the Baclite ${ }^{\mathrm{m}}$ assay, 14 results were defined as false negative and 40 as false positive. From these data, a diagnostic sensitivity of $71 \%$, a specificity of $82 \%$, positive and negative predictive values (PPV and NPV) of $47 \%$ and $95 \%$, respectively, were calculated from all sample results (Table I). Specificity, sensitivity, PPV and NPV for nares, chronic wounds and perineum were also calculated and given in Table I. The statistical performance of the test did not depend on the side of swabbing.

However, in 12 samples defined as MRSA negative by the reference methods, MRSA was confirmed, when the enriched Baclite ${ }^{\mathrm{Tx}}$ broth was subcultured onto MRSA ID agar. When these samples were included in the statistical performance of the Baclite ${ }^{\mathrm{Tm}}$ MRSA test, an overall sensitivity of $77 \%$ with a specificity of $87 \%$ was calculated for the new MRSA assay.

Hospitals and other health care facilities across the world are faced with alarming rates of infections caused by MRSA. Continuous spread of this pathogen requires efficient strategies for infection control, moreover since a 16-times higher transmission rate was suggested for MRSA carriers which are not subjected to contact isolation (Jernigan et al., 1996). However, conventional screening methods - as shown in the present study - require prolonged incubation and confirmatory testing up to 48 hours. During this time MRSA negative patients may be held in unnecessary isolation, whereas unidentified MRSA-positive individuals remain a hidden reservoir for cross-infection. To reduce the time taken for this evaluation, wards were selected (e.g. patients with previously reported MRSA in the last 3 months, chronic ulcers, antibiotic use in the last month) thus increasing the apparent prevalence of MRSA in the hospital. In this context, rapid identification or exclusion of MRSA colonization is essential for the effective control of MRSA. The majority of MRSA screening is carried out in clinical microbiological laboratories using culture based methods with or without prior broth enrichment. Broth based enrichment media enhance test sensitivity (Nahimana et al., 2006; Nonhoff et al., 2009), but adds an extra day to testing. As in the present study, the chromogenic MRSA ID agar medium supplemented with $4 \mathrm{mg}$ of cefoxitin/L is a widely used screening medium for MRSA. Although there is no one solid medium that is clearly superior, MRSA ID has demonstrated specificities and sensitivities of $>90 \%$ when compared to mecA PCR (Perry et al., 2004; Reverdy et al., 2005). Since an additional broth enrichment was used, the sensitivity and specificity of the reference methods is suggested to be similar to PCR methods.

Compared to the reference methods, a high NPV (95\%) of the Baclite ${ }^{\mathrm{max}}$ Rapid MRSA test was obtained allowing negative results to be confidently reported within $5 \mathrm{~h}$. In this view, our results are in line with the assay evaluation by Johnson et al. (2006) reaching a NPV of $98.7 \%$ for nasal screening swabs using mannitol salt agar plates containing oxacillin (MSAO agar) as a reference method. The diagnostic specificity and sensitivity for nasal swabs in our study was given with 70 and $84 \%$, respectively, and therefore lower $(94.6 \%$ and $96.9 \%$, respectively) as published by O'Hara et al. (2007). However, as negative samples make up the vast majority of MRSA screening tests particular in wards with a low MRSA prevalence, the high NPV evaluated for the Baclite $e^{\text {rx }}$ assay might represent a significant benefit to laboratories and the hygiene management. In contrast, in clinical settings with a high MRSA pressure, screening methods with a higher sensitivity and PPV should be used.

A useful feature of the assay is that it has been designed to retain a sample of the broth containing enriched MRSA from which to perform direct sensitivity tests and confirm presumptive positive or negative results. However, there were 12 Baclite $^{\mathrm{mw}}$ MRSA positive samples confirmed by MRSA ID from the enriched selective Baclite ${ }^{\text {mit }}$ broth, which were negative by the reference method. Although they were classified as "false positive" as the results were distinct from the reference method, they could not be considered as false positive in the usual sense of the term. When these samples were included in the statistical performance of the Baclite ${ }^{\mathrm{Tm}}$ MRSA test, an overall sensitivity of $77 \%$ with a specificity of $87 \%$ was calculated for the new MRSA assay. The better performance of the Baclite ${ }^{\mathrm{Tw}}$ test in these samples compared to the reference might be due to two major 
reasons: 1) an overgrowth of commensal organisms on the selective reference broth might mask the presence of MRSA as also discussed with regard to the selective MSAO agar used by Johnson et al. (2006). 2) Distinctive quality of swabbing could also be reasonable for test variations when two methods are compared (Kljakovic, 1992; Kingsley and Winfield-Davies, 2003).

As observed by Johnson et al. (2006) ciprofloxacin sensitivity of Staphylococcus aureus usually found in community acquired MRSA (CA-MRSA) could be a reason for the failed detection by the Baclite ${ }^{\mathrm{Tx}}$ assay. However, the frequency of CA-MRSA was $1.74 \%$ of all MRSA isolates in a German study, and therefore far less than reported from the USA (Witte et al., 2007). In general, ciprofloxacin supplemented medium seem to be a useful method for the detection of ciprofloxacin resistant MRSA. As shown by Davies and Zadik (1996), ciprofloxacin $(8 \mathrm{mg} / \mathrm{L})$ supplemented Baird-Parker medium (BPC) demonstrated a higher sensitivity for selection of MRSA than methicillin-supplemented mannitol salt agar (MMSA).

The material costs of the Baclite ${ }^{\mathrm{TM}}$ are near to $15 \mathrm{EURO}$ per single test, which is higher than a culture based method (around 1 EURO), but lower than commercial molecular based tests (>20 EURO) (Tacconelli et al., 2009). The Baclite ${ }^{\mathrm{TM}}$ test requires a relative low level of expertise and can be performed by a trained laboratory assistant, whereas the skill mix required to operate a PCR system may not be readily available in the diagnostic laboratory.

Taken together, we report a non molecular MRSA screening test, which is useful for the detection of MRSA from nares, throat, chronic wounds and perineum within $5 \mathrm{~h}$ and retains the advantages of a culture-based method. However, since the test has a high NPV of 95\% but is less sensitive and specific for the detection of ciprofloxacin resistant MRSA, the Baclite ${ }^{\mathrm{TM}}$ Rapid MRSA test seems to be more useful for wards with a low MRSA prevalence.

\section{Literature}

Davies S. and P.M. Zadik. 1997. Comparison of methods for the isolation of methicillin resistant Staphylococuus aureus. J. Clin. Pathol. 50: 257-258.

Diederen B.M.W., M.L. van Leest, I. van Duijn, P. Wilemse, P.H.J. van Keulen and J.A. Kluytmans. 2006. Performance of MRSA ID, a new chomogenic medium for detection of methicillinresistant Staphylococcus aureus. J. Clin. Microbiol. 44: 568-588.

Francois P., D. Pitet, M. Bento, B. Pepey, P. Vaudaux, D. Lew and J. Schrenzel. 2003. Rapid detection of methicillin-resistant Stapyhlococcus aureus directly from sterile or non-sterile clinical samples by a new molecular assay. J. Clin. Microbiol. 41: 254-260.

Gurran C., M.G. Holliday, J.D. Perry, M. Ford, S. Morgan and K.E. Orr. 2002. A novel selective medium for the detection of methicillin-resistant Staphylococcus aureus enabling result reporting in under 24 h. J. Hosp. Infect. 52: 148-151.
Harbarth S., C. Masuet-Aumatell and J. Schrenzel. 2006. Evaluation of rapid screening and pre-emptive contact isolation for detecting and controlling methicillin-resistant Staphylococcus aureus in critical care: an interventional cohort study. Crit. Care 10: R25.

Huletsky A., R. Giroux, V. Rossbach, M. Gagnon, M. Vaillancourt, M. Bernier and F. Gagnon. 2004. New real time PCR assay for rapid detection of methicillin resistant Staphylococcus aureus directly from specimens. J. Clin. Microbiol. 42: 1875-1884.

Jernigan J.A, M.G. Titus, D.H. Gröschel, S. Getchell-White and B.M. Farr. 1996. Effectiveness of contact bisolation during hospital outbreak of methicillin-resistant Staphylococcus aureus. Am. J. Epidemiol. 143: 496-504.

Johnson G., M.R. Millar, S. Matthews, M. Skyrme, P. Marsh, E. Barringer, S.O'Hara and M. Wilks. 2006. Evaluation of BacLite ${ }^{\mathrm{Tm}}$ Rapid MRSA, a rapid culture based screening test for the detection of ciprofloxacin and methicillin resistant S. aureus (MRSA) from screening swabs. BMC Microbiology 6: 83-88.

Kingsley A.M. and A. Winfield-Davies. 2003. Audit of wound swab sampling: why protocols could improve practice. Prof. Nurse 18: 338-343.

Kljakovic M. 1992. The quality of a laboratory test in general practice: the throat swab example. N.Z. Med. J. 105: 355-357.

Kluytmans J. 2007. Control of methicillin-resistant Staphylococcus aureus (MRSA) and the value of rapid tests. J. Hosp. Infect. 65: Suppl. 2, 100-104.

Nahimana I., P. Francioli and D.S. Blanc. 2006. Evaluation of three chromogenic media (MRSA-ID, MRSA-select and CHROMagar MRSA) and ORSAB for surveillance cultures of methicillin-resistant Staphylococcus aureus. Clin. Microbiol. Infect. 12: 1168-74.

Nonhoff C., O. Denis, A. Brenner, P. Buidin, C. Thiroux, M. Dramaix and M.J. Struelens. 2009. Comparison of three chromogenic media and enrichment broth media for the detection of methicillin-resistant Staphylococcus aureus from mucocutaneus screening specimens: comparison of MRSA chromogenic media. Eur. J. Clin. Microbiol. Inf. Dis. 28: 363-9.

O'Hara S., S. Gregory and D. Taylor. 2007. Evaluation of the $3 \mathrm{M}^{\mathrm{mm}}$ Baclite $^{\text {tix }}$ Rapid MRSA test for the direct detection of MRSA from nasal and groin surveillance specimens. Abstract of the Annual Meeting of the Infectious Disease Society of America, San Diego, CA, A540, 162-163.

Perry J.D., A. Davies, L.A. Butterworth, A.L.J. Hopley, A. Nicholson and F. K. Gould. 2004. Development and evaluation of a chromogenic agar medium for methicillin-resistan)t Staphylococcus aureus. J. Clin. Micobiol. 4: 4519-4523.

Reverdy M.E., S. Orenga, J.M. Roche, S. Delorme and J. Etienne. 2005. Multiresistant bacteria screening: clinical evaluation of MRSA ID, a new chromogenic medium for the screening of methicillin-resistant Staphylococcus aureus. abstr. P1383, $15^{\text {th }}$ European Congress Clin. Microbiol. Infec. Dis. 2005, ECCMID Copenhagen Denmark Squirrel D.J., M.J. Murphy, R.L. Leslie and J.C.D. Green. 2002. A comparison of ATP and adenylate kinase as bacterial cell markers: correlation with agar plate counts pp. 115-.123. In: R.A. Stanley and L.J. Kricka (eds). 2002. Bioluminescence and chemiluminescence progress and current applications. John Wiley and Sons.

Tacconelli E, G. De Angelis, M.A. Cataldo, G. La Torre and R. Cauda. 2009. Rapid screening tests for methicillin-resistant Staphylococcus aureus at hospital admission: a systematic review and meta-analysis. Lancet Inf. Dis. 9: 546-54.

Von Eiff C., D. Maas, G. Sander, A.W. Friedrich, G. Peters and K. Becker. 2007. Microbial evaluation of a new growth based approach for rapid detection of methicillin resistant Staphylococcus aureus. J. Antimicrob. Chemother. 61: 1277-1280.

Witte W., C. Braulke and B. Strommenger. 2007. Methicillin resistant Staphylococcus aureus containing the Panton-Valentine leucocidin gene in Germany in 2005 and 2006. J. Antimicrob. Chemotherap. 60: $1258-1263$. 